\title{
BK virus associated nephropathy and severe pneumonia in a kidney transplanted adolescent with Schimke immune- osseous-dysplasia
}

\author{
Ali Düzova1, Bora Gülhan ${ }^{1}$, Rezan Topaloğlu1 ${ }^{1}$, Fatih Özaltın¹,2, Ali Bülent Cengiz ${ }^{3}$, \\ Ayşe Filiz Yetimakman ${ }^{4}$, Deniz Doğru ${ }^{5}$, Şafak Güçer ${ }^{6}$, Nesrin Beşbaş ${ }^{1}$ \\ Department of Pediatrics ${ }^{1}$ Division of Pediatric Nephrology, ${ }^{2}$ Nephrogenetics Laboratory, ${ }^{3}$ Division of Pediatric Infectious \\ Diseases, ${ }^{4}$ Division of Pediatric Intensive Care, ${ }^{5}$ Division of Pediatric Pulmonary Medicine, ${ }^{6}$ Department of Pediatric and \\ Perinatal Pathology Research, Hacettepe University Faculty of Medicine, Ankara, Turkey. E-mail: aduzova@ hacettepe.edu.tr \\ Received: 1st August 2017, Accepted: 17th September 2017
}

SUMMARY: Düzova A, Gülhan B, Topaloğlu R, Özaltın F, Cengiz AB, Yetimakman AF, Doğru D, Güçer Ş, Beşbaş N. BK virus associated nephropathy and severe pneumonia in a kidney transplanted adolescent with Schimke immune-osseous-dysplasia. Turk J Pediatr 2019; 61: 111-116.

Patients with juvenile onset Schimke immune-osseous-dysplasia (SIOD) have less severe symptoms and can survive in the second and third decade of life. We present an 18 year-old adolescent with juvenile onset SIOD who was diagnosed after renal transplantation and developed BK virus associated nephropathy (BKVAN) and severe pneumonia during follow-up. The patient developed nephrotic syndrome, unresponsive to immunosuppressives, at the age of 8 years. He had a history of meningitis, short stature, microcephaly, prominent ears, and bilateral cryptorchidism. A renal transplantation was performed at the age of 15 years. During follow-up, he suffered from leucopenia, urinary tract infections, herpes labialis, and candida esophagitis. Sanger sequencing of SMARCAL1 revealed a missense mutation on exon 11 $(\mathrm{R} 586 \mathrm{~W})$. A renal biopsy performed after a sharp increase in serum creatinine (without significant viremia) revealed BKVAN which responded to sirolimus monotherapy and cidofovir. Three months later, he suffered from productive cough and dyspnea with diffuse ground glass pulmonary infiltrates. His clinical situation deteriorated and non-invasive mechanical ventilation was started. Cidofovir $(2 \mathrm{mg} / \mathrm{kg})$ was re-started weekly for a possible BKV pneumonia with intravenous immunoglobulin. After 5 doses of cidofovir and intense antibiotic regime, his dyspnea resolved with stable graft functions. In our case; BKVAN, which developed without significant viremia, and possibly associated pneumonia were treated successfully with cidofovir and sirolimus monotherapy.

Key words: BK virus associated nephropathy, kidney transplant, Schimke immuneosseous-dysplasia, pneumonia, sirolimus.

Schimke immuno-osseous dysplasia (SIOD) is an autosomal recessive multisystemic osteochondrodysplasia characterized by spondyloepiphyseal dysplasia (SED), T cell immunodeficiency and nephrotic syndrome.1,2 SIOD is caused by mutations in SMARCAL1, which encodes a protein homologous to the sucrose nonfermenting type 2 (SNF2) family of chromatin remodeling proteins. $^{2}$
The loss of SMARCAL1 function causes DNA replication associated genomic instability and this contributes to systemic features of SIOD. ${ }^{3}$ SIOD can be divided into two forms according to the presentation of the symptoms. The infantile form arises in utero and results in mortality in five years. However, patients with juvenile onset have less severe symptoms than infantile form and can survive in the second and third decade of life. Patients with 
severe symptoms usually die due to cerebral infarction, severe infections and end stage renal disease (ESRD). ${ }^{4}$ Ehrich et al. ${ }^{5}$ showed renal transplantation as a therapeutic option for ESRD in SIOD. Infections are important cause of concern for this group of patients after transplantation and data on optimal immunosuppressive treatment is lacking. ${ }^{6}$

Among the infectious agents, BK virus (BKV), a member of Polyomaviridae family, is a common posttransplant opportunistic virus. BKV affects nearly $15 \%$ of renal transplant recipients in the first posttransplant year. Data about management options are limited and untreated BKV-associated nephropathy (BKVAN) will progress to allograft failure. ${ }^{7}$ In addition, BKV can also cause extrarenal system involvement like meningitis, encephalitis, retinitis, colitis and vasculitis. ${ }^{8}$ Among them BKV pneumonia is quite rare and results in a fatal outcome. ${ }^{9,10}$

Here we report a juvenile onset SIOD patient who was diagnosed after renal transplantation and developed BKVAN and severe pneumonia during follow-up.

\section{Case Report}

A 6-year-old boy, treated in a local hospital with the diagnosis of meningitis, was referred to our center for further evaluation for microcephaly and bilateral cryptorchidism. He was the first child of consanguineous parents and born at $32^{\text {nd }}$ gestational week after an uneventful pregnancy. Physical examination revealed short stature, microcephaly, prominent ears and bilateral cryptorchidism; cranial computerized tomography (CT), brain magnetic resonance imaging, plasma/urine aminoacid profiles and audiogram were normal. At the age of 8 , he was diagnosed as nephrotic syndrome subsequent to an upper respiratory tract infection. Complement 3 and complement 4 levels were within normal limits and antinuclear antibody (ANA) was negative. Renal biopsy revealed focal segmental glomerulosclerosis. He was unresponsive to prednisolone, cyclosporine-A and cyclophosphamide. During outpatient visits, leucopenia was noticed; and examination of bone marrow aspiration was normal. No mutation was found in all exons of NPHS2 and in exons 8 and 9 of WT1. He progressed to ESRD and a renal transplantation from living related donor with 1 mismatch was performed at the age of 15 years, following a CAPD period of 4.5 years. Immunosupression consisted of basiliximab as induction and tacrolimus, prednisolone and mycophenolate mofetil (MMF) as maintenance therapy. At the first posttransplant year, he suffered from leucopenia, two episodes of urinary tract

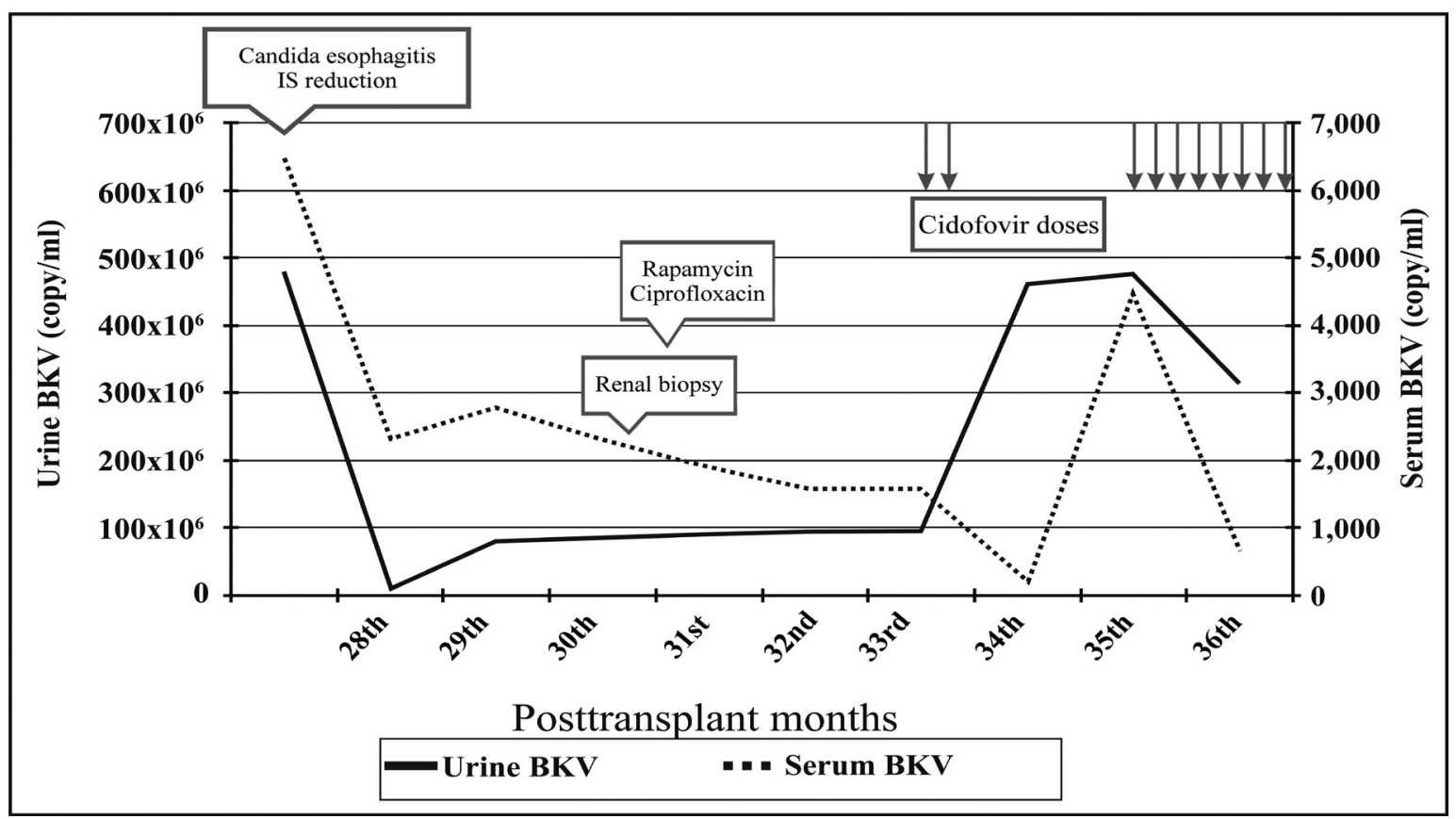

Fig. 1. Urine and serum BKV PCR levels and related therapies after renal transplantation. 
infection, and herpes labialis, with stable serum creatinine level. Serum and urine BKV and JC virus PCRs investigations were negative. At the second posttransplant year, he had oral ulcers several times and topical solutions were prescribed. His serum creatinine level was between $0.70-0.74 \mathrm{mg} / \mathrm{dl}$. At the beginning of the third posttransplant year, his serum creatinine level increased to $0.87-0.90 \mathrm{mg} /$ dl. DTPA scintigraphy suggested an acute rejection. Prednisolone dosage was increased and a renal biopsy was planned. Before the date of renal biopsy, he was admitted with the complaints of fever, back pain, dysphagia and loss of appetite with leukocyte count of $1,500 / \mathrm{mm}^{3}$ and absolute neutrophil count of $400 / \mathrm{mm}^{3}$. Endoscopy revealed white plaques in the esophagus and endoscopic biopsy showed proliferating candida hyphae on esophageal mucosa. Urine and serum PCR for BKV revealed BKV viruria without viremia (Fig. 1); serum CMV PCR (474 copy/ml) was also positive. Because of severe candida infection, the doses of prednisolone, tacrolimus and mycophenolate mofetil (MMF) were decreased. Renal biopsy could not be performed due to fever. With antimicrobial therapy and parenteral fluids, his creatinine level decreased $(0.73 \mathrm{mg} / \mathrm{dl})$ and his dyspeptic symptoms resolved. He was discharged after 20 days of fluconazole, 10 days of ganciclovir, 15 days of meropenem and ornidazole and 12 days of caspofungin. Three weeks later, his serum creatinine level was $0.73 \mathrm{mg} / \mathrm{dl}$ and BKV viruria was decreased (Fig. 1). However, 2.5 months later, he had a sharp increase in serum creatinine level $(1.02 \mathrm{mg} / \mathrm{dl})$. Renal biopsy revealed BKVAN (genotype 1).

On the basis of recurrent severe infections, leucopenia and history of renal involvement, SIOD was suspected. Sanger sequencing of SMARCAL1 revealed a missense mutation on exon 11 (R586W), that had been described previously. ${ }^{11}$ Skeletal radiography did not reveal any SED finding. CD4/CD8 ratio was decreased. Prednisolone, tacrolimus and MMF was stopped. Sirolimus monotherapy and cidofovir were initiated for BKVAN. After two doses of cidofovir ( $1 \mathrm{mg} / \mathrm{kg} /$ dose $)$ BKV serum PCR level was decreased and urine PCR level increased (Fig. 1).

Three months after the renal biopsy, he was
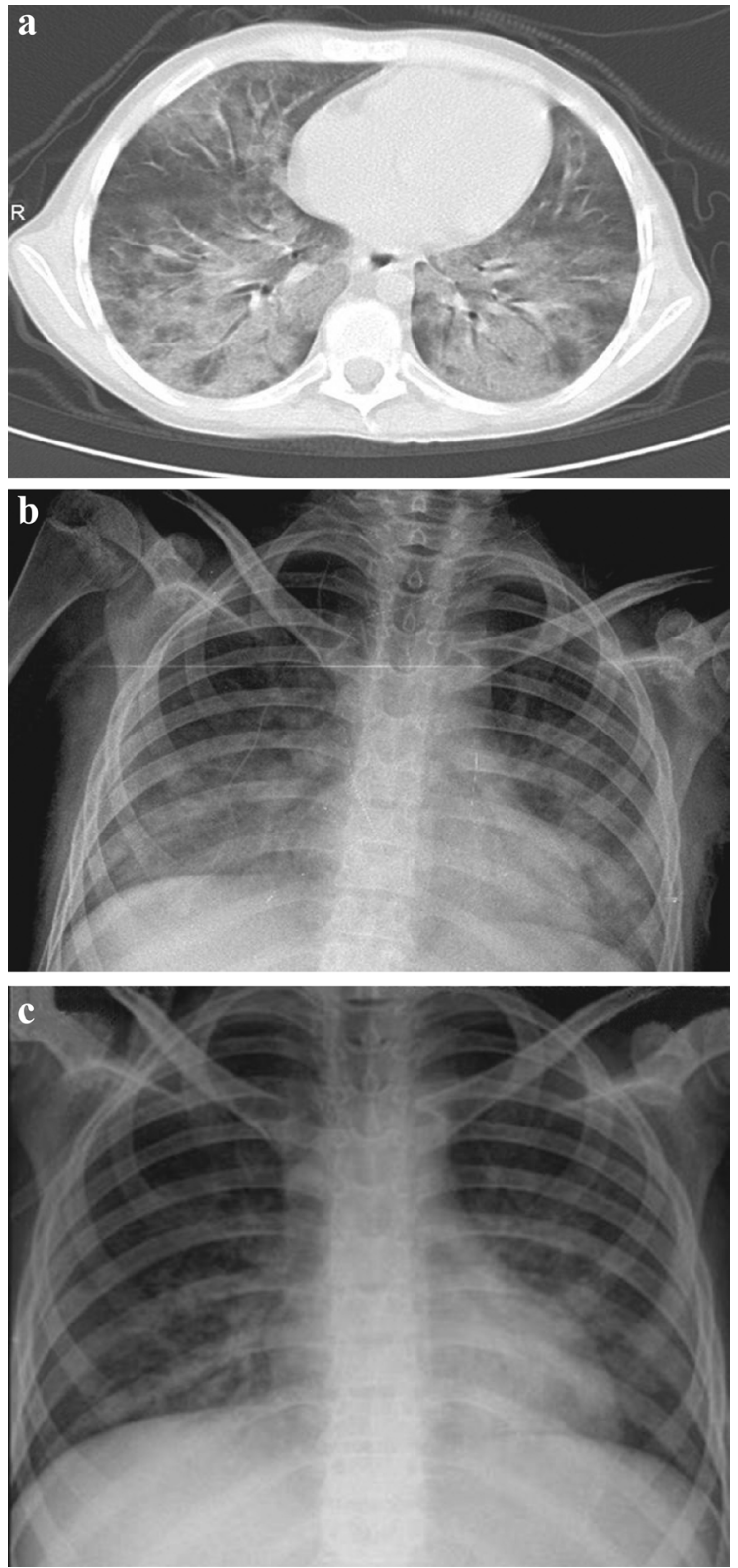

Fig. 2. Thorax CT and chest X-ray of the patient at admission for pneumonia ( $a, b)$ showing bilateral paracardiac infiltration and bilateral diffuse ground glass appearance more prominent on lower lobes; and chest X-ray after antibiotic treatment (c).

admitted with complaints of productive cough, dyspnea. On physical examination, he was tachypneic and dyspneic with perioral cyanosis and bilateral crackles on lower hemithorax. Chest X-ray showed bilateral paracardiac infiltration. Thorax CT showed diffuse ground glass appearance more prominent on lower lobes bilaterally (Fig. 2a). Meropenem, teicoplanin, ciprofloxacin, trimethoprim-sulphamethoxazole 
(TMP-SMX), amphotericin B and clarithromycin were initiated. Despite antibiotic and oxygen treatment, the clinical situation deteriorated and hypoxia did not resolve, therefore he was transferred to the Intensive Care Unit (ICU) for acute respiratory failure and non-invasive mechanical ventilation via full-face mask was started. Cidofovir was re-started weekly for a possible BKV pneumonia at a dose of $2 \mathrm{mg} / \mathrm{kg}$. Intravenous immunoglobulin (IVIG) treatment was also initiated every three weeks at a dose of $400 \mathrm{mg} / \mathrm{kg}$. Sirolimus through level was maintained between 3-5 ng/ml. After 5 doses of cidofovir and intense antibiotic regime, his dyspnea resolved. He was followed for 20 days in ICU with non-invasive mechanical ventilation and was discharged after 39 days of hospitalization. Hyperpigmented macules were noticed on the trunk. Control chest X-ray showed radiological improvement (Fig. 2b). Three additional cidofovir doses were administered during outpatient visits. Patient was maintained on TMP-SMX prophylaxis, IVIG (every three weeks) and sirolimus monotherapy with stable graft functions (creatinine $0.75 \mathrm{mg}$ / dl). Informed consent was received from the patient and his parents for this report.

\section{Discussion}

Recurrent and severe infections, vaso-occlusive processes, and ESRD are frequent causes of death in SIOD. Nephropathy progresses to ESRD and renal transplantation is the mainstay of the treatment. ${ }^{5}$ The type of mutation in SMARCAL1 affects the severity of SIOD. The infantile form of SIOD is caused by a deletion, nonsense or frame-shift mutation of SMARCAL1. On the other hand, missense mutations allow retention of partial SMARCAL1 function and cause a milder disease regardless of whether the second allele encodes a nonsense or missense mutation. ${ }^{2}$

Our patient was a juvenile onset SIOD; he developed ESRD at the age of 10.5 years and a homozygous missense R586W mutation was detected. Mutations of conserved arginine residues, R586W, R644W, R645C, $\mathrm{R} 764 \mathrm{Q}$ and $\mathrm{R} 820 \mathrm{H}$ were the most common missense mutations. R586W mutation was first described by Boerkoel et al. ${ }^{11}$ in 2002, in two Italian children (one male and one female) of consanguineous parents; they had
SED findings without dysmorphic facial features or hyperpigmented macules on the trunk; they had not suffered from recurrent infections or CNS involvement. Both cases had renal involvement, but detailed information was not available. Compared to Italian patients with R586W mutation, our case did not have SED findings; he had no dysmorphic features other than microcephaly and prominent ears; he developed hyperpigmented macules on the trunk at the age 17 years; and he had suffered from meningitis at the age of 6 years.

Clewing et al. $^{2}$ proposed a disease severity score (based on linear growth failure before 10 years of age, renal failure, lymphopenia, recurrent infections, cerebral ischemia, age at death; with a maximum score of 7) for different types of SMARCAL1 mutations. Italian patients were scored as 2 . The score of our patient was 4 , indicating a more severe course. As suggested by Clewing et al. ${ }^{2}$, genotypephenotype correlation does not extend to individual patients and cannot be used as a prognostic indicator.

Lücke et al. ${ }^{6}$ reported renal transplantation experience in a group of SIOD patients and investigated recurrent infection status before and after renal transplantation. They stated that $25-30 \%$ of SIOD patients had an increased frequency of severe infections following renal transplantation. One of their patients carried homozygous R586W mutation. This patient developed renal failure at the age of 11 years and a renal transplantation was performed at the age of 23 years; primary immunosuppressive regime consisted of azathioprine, cyclosporine $\mathrm{A}$ and prednisone; the type of infections was not changed with this regime. However, our patient with same mutation suffered from candida esophagitis, BKVAN and severe pneumonia after renal transplantation when he was under similar immunosuppressive regime consisting of MMF, tacrolimus and prednisone, which may suggest the presence of epigenetic factors that might modify the course of the disease.

In the context of SIOD based-immunodeficiency, optimal immunosuppressive regime is still a matter of debate. Lücke et al. ${ }^{6}$ showed, for the first time, the efficacy of immunosuppressive monotherapy in 5 posttransplant children with SIOD. In two patients, renal function was improved with MMF monotherapy without graft 
rejection. In other three patients (tacrolimus, cyclosporine, and sirolimus monotherapy, respectively), renal function remained stable. In the latter group, similar to our case, one of the patients continued with sirolimus monotherapy. After start of sirolimus monotherapy, she did not have any infection for two years. In contrast, our patient experienced severe pneumonia under sirolimus monotherapy.

In our patient, one of the main reasons for sirolimus conversion was the development of BKVAN, which is another threat for transplanted children. Although it is ubiquitous in adults with a seroprevalence of $>80 \%$, it becomes pathogenic in renal transplant recipients. American Society of Transplantation defines a BK viral load of 10,000 copies $/ \mathrm{ml}$ for $>3$ weeks as presumptive BKVAN and recommends reduction in immunosuppression. ${ }^{12}$ However, Hassan et al. ${ }^{13}$ showed that cut-off level of 10,000 copies/ $\mathrm{ml}$ underestimated the diagnosis of BKVAN. Consistent with this; in our patient, all serum BKV titers were below 10,000 copies/ $\mathrm{ml}$, despite a clear evidence of BKVAN in biopsy.

A number of risk factors for development of BKVAN related to donor, recipient and transplantation have been proposed in different studies. The most consistent risk factor is the overall degree of immunosuppression. In immunological system, $T$ cell immunity is implicated in fighting against BKV. Absolute CD4 count is inversely proportional to urinary BKV replication. ${ }^{8}$ In our patient, $T$ cell immunodeficiency secondary to SIOD and immunosuppressive treatment were two risk factors for BKVAN. The mainstay of the BKVAN treatment is the reduction of immunosuppression. The most common approach is the discontinuation of antimetabolite. But tacrolimus and cyclosporine can inhibit anti-BK $\mathrm{T}$ cell responses in vitro. ${ }^{14}$ Therefore, dose reduction of calcineurin inhibitor (CNI) targeting lower through levels is also recommended. In addition to dose reduction, cidofovir, leflunomide, ciprofloxacin, rapamycine and IVIG are other treatment alternatives. A meta-analysis of series using cidofovir, leflunomide or IVIG reported similar clearance rates for each agent. ${ }^{15}$ In our patient, in addition to dose reduction of immunosuppressive drugs, sirolimus monotherapy was preferred due to immunodeficiency secondary to SIOD.

After the diagnosis of BKVAN, our patient developed severe respiratory symptoms, suggestive of BKV related pneumonitis which usually has a fatal outcome. There are less than 10 patients with BKV associated pneumonia in the literature. Sandler et al. ${ }^{16}$ reported an 8-month-old girl with osteopetrosis. She developed hemorrhagic cystitis on day-3. On day-19, her respiratory status was worsened despite invasive respiratory support and she died. BKV was isolated from cell culture of the lung tissue and bladder. Cubukcu-Dimopulo et al. ${ }^{17}$ reported a 14 -year-old boy who was followed with the diagnosis of AIDS since the age of 10 years. He had been admitted to the hospital several times for recurrent and severe infections. At last hospitalization, transbronchial lung biopsy was taken. $\mathrm{He}$ developed severe respiratory distress and died at $5^{\text {th }}$ day of admission. Cidofovir was not given in the two aforementioned patients. Adult patients with underlying malignancy were also reported to have BKV pneumonia and they only survived less than 7 days. ${ }^{9,10}$

The first BKV-pneumonitis case that was diagnosed in antemortem period was reported by Yapa et al. ${ }^{18}$. It was a 19-year-old male who underwent allogeneic HSCT for high risk AML. Cells infected with BKV were seen in cytopathological examination of bronchoalveolar lavage (BAL). His treatment for BKV consisted of weekly cidofovir $(2.5 \mathrm{mg} / \mathrm{kg} / \mathrm{dose})$ and IVIG. The patient did not respond to treatment and died due to multiorgan failure. In our patient, pneumonia could have been due to BKV or other respiratory viruses, bacteria or other fungal and opportunistic pathogens as he was an immunocompromised patient. Unfortunately, we could not perform a BAL or lung biopsy to demonstrate the microorganism responsible for his pneumonia due to his unstable clinical status. Besides, antimicrobial therapy was already started and included treatment against a broad range microorganism including fungal and opportunistic pathogens (e.g. P. jirovecii). Because of the aforementioned reasons, clinical improvement in our case could not be attributed to cidofovir doses and IVIG treatment, solely. In conclusion, recurrent and severe infections following renal transplantation may be 
suggestive of immune deficiency with mild forms; and SIOD should be considered for differential diagnosis. BKVAN can develop without significant viremia; renal biopsy should be considered in such cases. Posttransplant SIOD patients should be followed very closely and in case of BKVAN, the management should include an aggressive approach. In our case, BKVAN and possibly associated pneumonia were treated successfully with cidofovir and reduction of immunosuppressive drugs.

\section{REFERENCES}

1. Schimke RN, Horton WA, King CR. Chondroitin-6sulfaturia, defective cellular immunity, and nephrotic syndrome. Lancet 1971; 2: 1088-1089.

2. Clewing JM, Fryssira H, Goodman D, et al. Schimke immunoosseous dysplasia: Suggestions of genetic diversity. Hum Mutat 2007; 28: 273-283.

3. Sarin S, Javidan A, Boivin F, et al. Insights into the renal pathogenesis in Schimke immuno-osseous dysplasia: A renal histological characterization and expression analysis. J Histochem Cytochem 2015; 63: 32-44.

4. Lou S, Lamfers P, McGuire N, Boerkoel CF. Longevity in Schimke immune-osseous dysplasia. J Med Genet 2002; 39: 922-925.

5. Ehrich JH, Offner G, Schirg E, Hoyer PF, Helmchen $\mathrm{U}$, Brodehl J. Association of spondylo-epiphyseal dysplasia with nephrotic syndrome. Pediatr Nephrol 1990; 4: 117-121.

6. Lücke T, Kanzelmeyer N, Baradaran-Heravi A, et al. Improved outcome with immunosuppressive monotherapy after renal transplantation in Schimkeimmuno-osseous dysplasia. Pediatr Transplant 2009; 13: $482-489$.

7. Sawinski D, Goral S. BK virus infection: An update on diagnosis and treatment. Nephrol Dial Transplant 2015; 30: 209-217.
8. Siguier M, Sellier P, Bergmann JF. BK-virus infections: A literature review. Med Mal Infect 2012; 42: 181-187.

9. Galan A, Rauch CA, Otis CN. Fatal BK polyoma viral pneumonia associated with immunosuppression. Hum Pathol 2005; 36: 1031-1034

10. Akazawa Y, Terada Y, Yamane T, et al. Fatal BK virus pneumonia following stem cell transplantation. Transpl Infect Dis 2012; 14: E142-E146.

11. Boerkoel CF, Takashima H, John J, et al. Mutant chromatin remodeling protein SMARCAL1 causes Schimke immuno-osseous dysplasia. Nat Genet 2002; 30: 215-220.

12. Hirsch HH, Randhawa P. AST Infectious Diseases Community of Practice. BK virus in solid organ transplant recipients. Am J Transplant 2009; 9(Suppl 4): S136-S146.

13. Hassan S, Mittal C, Amer S, et al. Currently recommended BK virus (BKV) plasma viral load cutoff of $\geq 4 \log 10 / \mathrm{mL}$ underestimates the diagnosis of BKV-associated nephropathy: A single transplant center experience. Transpl Infect Dis 2014; 16: 55-60.

14. Egli A, Köhli S, Dickenmann M, Hirsch HH. Inhibition of polyomavirus BK-specific T-cell responses by immunosuppressive drugs. Transplantation 2009; 88 : 1161-1168.

15. Hilton R, Tong CY. Antiviral therapy for polyomavirusassociated nephropathy after renal transplantation. J Antimicrob Chemother 2008; 62: 855-859.

16. Sandler ES, Aquino VM, Goss-Shohet E, Hinrichs $\mathrm{S}$, Krisher K. BK papova virus pneumonia following hematopoietic stem cell transplantation. Bone Marrow Transplant 1997; 20: 163-165.

17. Cubukcu-Dimopulo O, Greco A, Kumar A, Karluk D, Mittal K, Jagirdar J. BK virus infection in AIDS. Am J Surg Pathol 2000; 24: 145-149.

18. Yapa HM, McLornan DP, Raj K, et al. Pneumonitis post-haematopoeitic stem cell transplant-cytopathology clinches diagnosis. J Clin Virol 2012; 55: 278-281. 